Check for updates

Cite this: J. Mater. Chem. B, 2021 9, 2623

Received 6th December 2020,

Accepted 3rd February 2021

DOI: $10.1039 / d 0 t b 02833 a$

rsc.li/materials-b

\section{Near-infrared fluorescent probe for evaluating the acetylcholinesterase effect in the aging process and dietary restriction via fluorescence imaging $\dagger$}

\author{
$\mathrm{Na} \mathrm{He}{ }^{a}$ Lei $\mathrm{Yu},{ }^{\mathrm{D}}{ }^{\mathrm{b}}$ Minghua $\mathrm{Xu},{ }^{\mathrm{C}}$ Yan Huang, de Xiaoyan Wang, de \\ Lingxin Chen (iD *def and Shouwei Yue*a
}

\begin{abstract}
Dietary restriction (DR), as a natural intervention, not only benefits the neuroendocrine system, but also has an antiaging action. Acetylcholinesterase (AChE) is one of the most important bioactive substances and plays a major part in choline changes in the aging process. Thus, we aim to evaluate the effect of DR on AChE in the brains of aging animals. In this study, we synthesize a NIR fluorescent probe $\mathrm{BD}-\mathrm{AChE}$ for the real-time and in situ monitoring of AChE level changes in living cells and living mice, notably in brains. In situ visualization with BD-AChE verified a decrease in the AchE level in the brains of mice aging models. Evidently, the prepared probe has the excellent capability of measuring AChE variation in the brains of aging mice with DR via NIR fluorescence bioimaging, indicating that long-term DR can effectively affect AChE levels in the brain. The attenuation of AChE level in the brain of aging mice after DR could be helpful in infering the advantageous impact of DR on age-related neurodegenerative disease, as a better treatment alternative in the future.
\end{abstract}

\section{Introduction}

The world's population is aging rapidly. Aging, an inescapable biological process, has been defined as the gradual accumulation of various harmful changes with time which increases the chance of illness and death. Many neurotransmitter systems appear to be compromised during human aging. ${ }^{1}$ The cholinergic system is the main neurotransmitter system, which is responsible for cognitive symptoms in normal aging. The aging-related decline in cholinergic function of the central nervous system is considered to be one of the causes for short-term memory impairment during the aging process. ${ }^{2}$ The cholinergic hypothesis of senile memory dysfunction raised by Bartus assumes that there are functional disorders

\footnotetext{
${ }^{a}$ Rehabilitation Center, Qilu Hospital, Cheelo College of Medicine, Shandong University, Jinan 250100, China. E-mail: shouweiy@sdu.edu.cn

${ }^{b}$ Shandong Peninsula Engineering Research Center of Comprehensive Brine Utilization, Weifang University of Science and Technology, Weifang 262700, China

${ }^{c}$ Yantai Central Blood Station, Yantai 264003, China

${ }^{d}$ CAS Key Laboratory of Coastal Environmental Processes and Ecological Remediation, Yantai Institute of Coastal Zone Research (YIC), Chinese Academy of Sciences (CAS), Shandong Key Laboratory of Coastal Environmental Processes, YICCAS, Yantai 264003, China. E-mail: lxchen@yic.ac.cn

${ }^{e}$ School of Pharmacy, Binzhou Medical University, Yantai, 264003, China

${ }^{f}$ Center for Ocean Mega-Science, Chinese Academy of Sciences, Qingdao 266071, China

$\dagger$ Electronic supplementary information (ESI) available. See DOI: 10.1039/ dotb02833a
}

in the cholinergic activity in the brains of patients with dementia and healthy older people and that these functional disorders play a vital role in related cognitive problems and memory loss. Therefore, recovery of cholinergic function may relieve the cognitive loss. ${ }^{3,4}$ This hypothesis has been strongly supported by the fact that acetylcholinesterase (AChE) inhibitors show positive therapeutic effects on cognition in people with Alzheimer's disease (AD). Since the initial proposal, many studies on the clinical development of cholinergic agents have followed, and though the overall clinical efficacy is limited, cognitive enhancers for modulating cholinergic function are still one of the most widely used drugs which have been approved for $\mathrm{AD} .{ }^{5}$ Due to its ability to rapidly hydrolyze the neurotransmitter acetylcholine into choline and acetate, AChE makes it possible to control the precise time of synaptic activation. Therefore, the biological activity of AChE is the key marker for cholinergic metabolism. AChE is also closely related to brain growth, learning, memory and nerve injury, besides participating in cholinergic transmission. ${ }^{6}$

Dietary restriction (DR), as a method of reducing calorie intake without malnutrition, has been widely believed to affect multiple physiological events, involving the neuroendocrine signalling systems. ${ }^{7}$ It modifies protein turnover, changes the expression of numerous genes, and reduces the glycosylation of macromolecules and has been proved to enhance the function of synaptic plasticity, including learning ability and long-term potentiation. ${ }^{8,9}$ In previous studies, the rats that maintained 
DR performed better on memory and learning tasks than the age-matched rats that were fed ad libitum. ${ }^{10}$ Relying on the capability of prompting the effective expression of neurotrophic factors and particular gene encoded proteins which facilitate synaptic plasticity and neuronal survival, DR has great benefits for the nervous system. ${ }^{11}$ Importantly, it has anti-aging action; the mild nutritional stress stimulated by DR might effectively induce metabolic response to provide better survivability and extend lifespan. ${ }^{12}$

From what has been discussed above, AChE plays a very significant part in the choline changes in the aging process. $\mathrm{DR}$, as a mild intervention, not only has beneficial effects on the neuroendocrine system, but also has antiaging action. We aim at assessing the underlying effect of DR on AChE in the brains of aging animals. Thus, an efficient tool for detecting the AChE level can be more helpful in exploring the connection between DR and AChE. Until now, there have been several methods for detecting AChE, such as the colorimetric Ellman method or the detection of hydrogen peroxide generated by choline oxidation. ${ }^{13}$ In addition, most of them detect AChE according to other biomolecules. Due to the low sensitivity, and poor indirectness and specificity of these methods, they cannot be well applied to the analysis of biological samples. ${ }^{14-17}$ The bioimaging technology coupled with small molecule fluorescent probes has the outstanding advantages of high spatio-temporal resolution, in situ and real-time detection, high sensitivity and specificity, as well as non-invasive and rapid analysis, and has been developed into an excellent technology for examining biological species in biosystems. ${ }^{18-21}$ Thus, small molecule fluorescent probes can accurately assess the level of a biological enzyme while sustaining the activity of the biological enzyme in living systems. $^{22}$ Since AChE plays a significant role in the pathological and physiological processes, a number of researchers have been committed to designing and synthesizing fluorescent probes for the detection of AChE in biological systems. ${ }^{23-33}$ However, in view of the fact that the excitation and emission wavelengths of these probes are located in the visible region, bioimaging is frequently hindered by background fluorescence, which makes them not suitable for imaging deep tissues. Therefore, it is urgently necessary to design and synthesize an ideal and appropriate NIR fluorescent probe for the detection and imaging of AChE in living cells and animals.

In this study, we generated a desirable probe BD-AChE as a chemical bioimaging tool to image and detect AChE in living cells and in vivo. BD-AChE was capable of providing excellent selectivity and sensitivity to AChE in a biological environment. The experimental results validated that the AChE levels decreased in the brain of mice aging models. We also indicated that the AChE levels in the brains of aging mice significantly decreased after DR. To the best of our knowledge, BD-AChE is the first fluorescent probe for the imaging of AChE in old DR mice, and provides clear evidence on the connection between the AChE level and DR in aging mice. These results help to infer the beneficial effects of DR on age-related neurodegenerative diseases as a better treatment option in the future.

\section{Experimental}

\subsection{Synthesis of BD-AChE}

Cesium carbonate $(0.326 \mathrm{~g}, 1 \mathrm{mM})$ and compound $4(0.529 \mathrm{~g}$, $1 \mathrm{mmol}$ ) were mixed in dichloromethane. The mixture was stirred at room temperature under argon for $30 \mathrm{~min}$; then, we added dimethylcarbamoyl chloride to the compound $(400 \mu \mathrm{L})$. Next, the compound was stirred for 3 days and dimethyl methotrexate $(200 \mathrm{~L})$ was added twice a day. By performing column chromatography on silica with $\mathrm{CH}_{2} \mathrm{Cl}_{2} / \mathrm{CH}_{3} \mathrm{OH}(10: 1)$ as the eluent, the compound was purified, and we obtained a yellow product $(0.395 \mathrm{~g}, 59 \%) .{ }^{1} \mathrm{H}$ NMR $\left(500 \mathrm{MHz}, \mathrm{CD}_{3} \mathrm{OD}-\mathrm{D}_{4}\right) \delta$ (ppm): 8.16-8.14 (m, 1H), 8.06-8.04 (m, 2H), 7.88-7.80 (m, 2H), 7.51-6.92 (m, 13H), 4.85 (s, 12H), 2.97-2.84 (m, 2H). ${ }^{13} \mathrm{C}$ NMR (125 MHz, $\left.\mathrm{CD}_{3} \mathrm{OD}-\mathrm{D}_{4}\right) \delta$ (ppm): 166.1, 163.5, 158.6, 157.7, 157.4, 150.3 , 139.5, 138.7, 130.8, 129.5, 129.0, 128.9, 128.8, 128.7, $128.6,128.3,128.2$, 128.1, 127.9, 127.6, 126.8, 121.7, 115.8, 115.3, 115.1, 37.5. LC-MS $\left(\mathrm{ESI}^{+}\right): m / z \quad \mathrm{C}_{38} \mathrm{H}_{32} \mathrm{BF}_{2} \mathrm{~N}_{5} \mathrm{O}_{4}$, calcd 671.2515, $[\mathrm{M}+\mathrm{H}]^{+} 672.2468$.

\subsection{Animals and diet}

In this research, we utilized female Swiss albino (Balb/C) mice as the research objects. The mice kept in polycarbonate cages were reared under normal laboratory conditions $\left(25 \pm 2{ }^{\circ} \mathrm{C}\right.$; $12 \mathrm{~h}$ light/dark cycle), and fed with normative water and pellet feed according to the established experimental plan. To determine the potential relationship between normal activity of AChE and age, we selected four distinct age groups (1, 6, 12, and 18 months). The laboratory procedures of animals were performed in accordance with the institutional regulations for laboratory animal use and care. All mice experimental procedures were performed in conformity with the institutional regulations and guidelines for the use and care of laboratory animals, and the related protocols were approved by the Institutional Animal Care and Use Committee in Binzhou Medical University, Yantai, China. Approval Number: No. BZ2014-102R.

\subsection{Fasting regimen}

We divided the 18 month-old mice in this study into two groups according to the experimental protocol. The first group was the control group. The mice in the first group were fed freely, and they were provided with a continuous food supply. The mice in the second group were made to fast for 24 hours. It should be noted that water was periodically provided to all experimental mice.

\subsection{Dietary restriction regimen}

We divided the 18 month-old mice in this study into four distinct groups according to the experimental plan. The first group was the control group. The mice in control group were fed freely, and they were provided with a continuous food supply. The mice in the DR regimen group were given food on alternate days for 1, 2 and 3 months, separately. It should be noted that water was periodically provided to all experimental mice. 


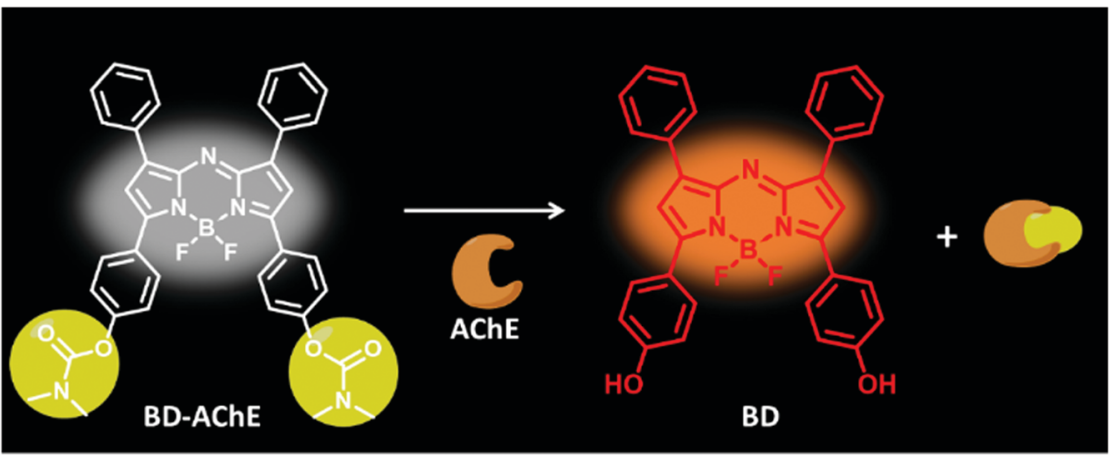

Scheme 1 Detection mechanism of BD-AChE towards AChE.

\section{Results and discussion}

\subsection{Design of probe $\mathrm{BD}-\mathrm{AChE}$}

The chemical synthetic procedure for BD-AChE is clearly exhibited in Schemes S1 and S2 (ESI $\dagger$ ). The details of chemical synthesis of various compounds are described in the $\mathrm{ESI} \dagger$ of this article. The reaction mechanism of BD-AChE is displayed in Scheme 1. The various compounds shown in this article were characterized using ${ }^{1} \mathrm{H}$ NMR, ${ }^{13} \mathrm{C}$ NMR and HRMS. We used a BODIPY dye to serve as a fluorophore unit for emitting a fluorescence signal, and its emission wavelength was located in the NIR range. Given that tissue auto-fluorescence and absorption are very low in the NIR region, optical bioimaging with an NIR fluorophore exhibits better penetration of deep tissues and higher sensitivity. In this study, in order to acquire an excellent probe for detecting AChE, the particular recognition moiety of the dimethyl carbamate group was merged into the fluorophore unit. The dimethyl carbamate group can bind covalently to a serine residue in the active site of AChE, then cause the carbamylation of serine in the active site of AChE, resulting in the deactivation of AChE. The carbonyl group of BD-AChE is bound to the oxygen atom, the emission of BODIPY was quenched by the dimethyl carbamate group due
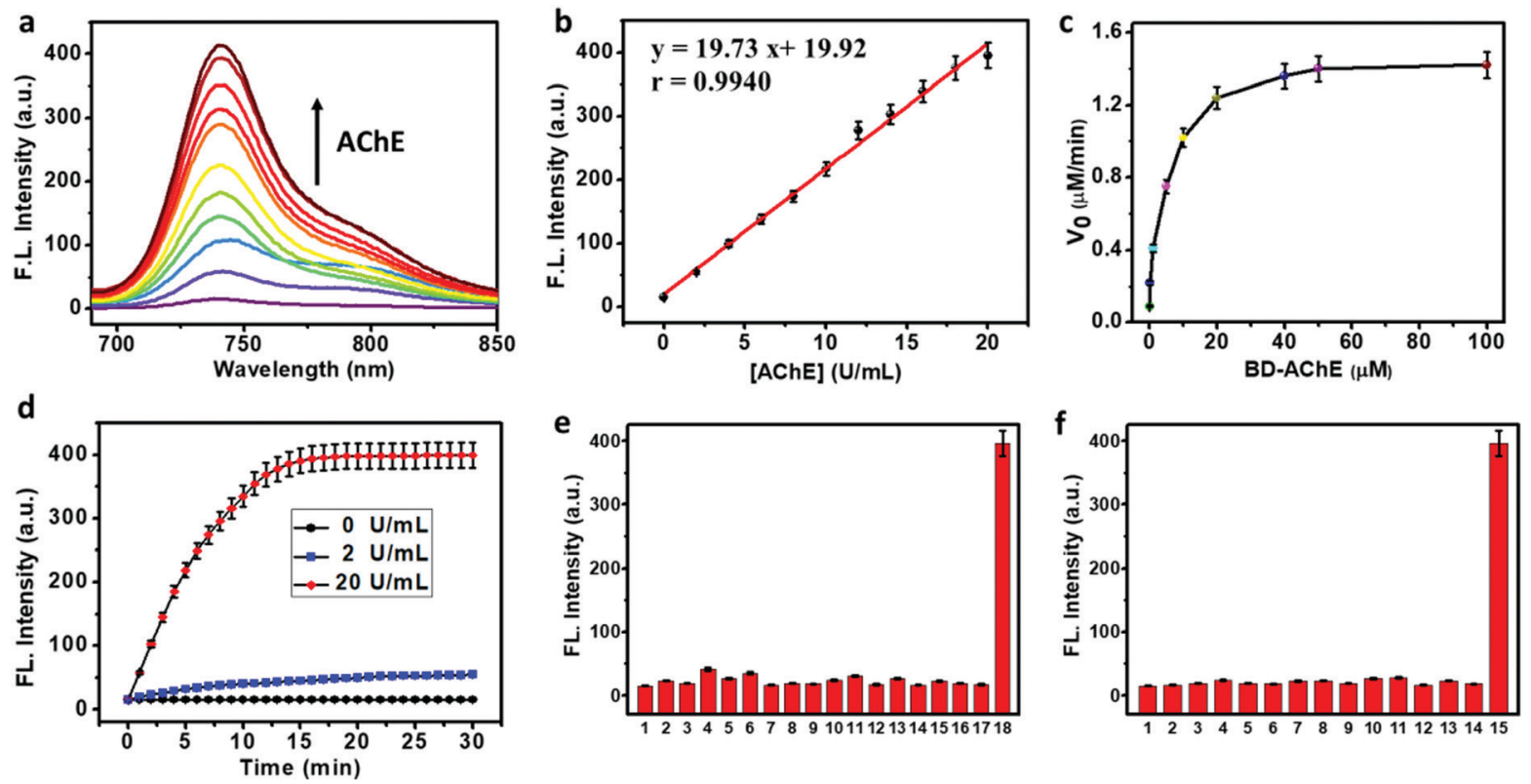

Fig. 1 Spectral properties and enzymatic properties of BD-AChE. (a) Variations in the fluorescence emission spectra of BD-AChE (10 $\mu M)$ with a wide range of concentrations of $\mathrm{AChE}\left(0,2.0,4.0,6.0,8.0,10.0,12.0,14.0,16.0,18.0\right.$ and $20.0 \mathrm{U} \mathrm{mL} \mathrm{m}^{-1}$ ). (b) The plot of the linear relationship between the relative fluorescence intensity at $740 \mathrm{~nm}$ and $A C h E$ concentration $\left(0-20.0 \mathrm{U} \mathrm{mL}^{-1}\right.$ ). (c) Michaelis-Menten plot of the reaction between different concentrations of BD-AChE $(0.05-100 \mu \mathrm{M})$ and $20.0 \mathrm{U} \mathrm{mL}^{-1} \mathrm{AChE}$. (d) Plot of fluorescence intensity of BD-AChE $(10 \mu \mathrm{M})$ against the reaction time with various $A C h E$ concentrations $\left(0,2\right.$, and $\left.20 \mathrm{U} \mathrm{mL}^{-1}\right)$. (e) Fluorescence responses of BD-AChE $(10 \mu \mathrm{M})$ towards various enzymes $2-10\left(1.0 \mu \mathrm{g} \mathrm{mL}{ }^{-1}\right)$, $11-17$ biospecies $(0.1 \mathrm{mM})$ and $\mathrm{AChE}\left(20.0 \mathrm{U} \mathrm{mL} \mathrm{mL}^{-1}\right.$ ) after being mixed for $15 \mathrm{~min}$ : (1). Blank, (2). butyrylcholine esterase, (3). pepsin, (4). peroxidase, (5).horseradish, (6). glutathione S-transferases, (7). nitroreductase, (8). monoamine oxidase A, (9). monoamine oxidase B, (10) alkaline phosphatase, (11). BSA, (12). glucose, (13). cysteine, (14). alanine, (15). glycine, (16). lysine, (17). arginine and (18). AChE. (f) Fluorescence responses of BD-AChE (10 $\mu$ M), towards $100 \mu \mathrm{M}$ of a variety of anions and metal ions (1). Blank, (2). $\mathrm{Na}^{+}$, (3). $\mathrm{K}^{+},(4) \cdot \mathrm{Zn}^{2+},(5) \cdot \mathrm{Mg}^{2+},(6) . \mathrm{Ca}^{2+},(7) \cdot \mathrm{Cl}^{-},(8) . \mathrm{Br}^{-},(9) . \mathrm{HSO}_{3}^{-},(10) . \mathrm{SO}_{4}^{2-},(11) \cdot \mathrm{SO}_{3}^{2-},(12)$. $\mathrm{S}_{2} \mathrm{O}_{3}^{2-}$, (13). $\mathrm{CO}_{3}^{2-}$, (14). $\mathrm{H}_{2} \mathrm{PO}_{4}^{2-}$, and (15). AChE. $\lambda_{\mathrm{ex}}=680 \mathrm{~nm}, \lambda_{\mathrm{em}}=740 \mathrm{~nm}$. The tests were carried out in HEPES $\left(10 \mathrm{mM}, \mathrm{pH} 7.4,37^{\circ} \mathrm{C}\right)$. 
to the photoninduced electron-transfer (PET) mechanism; thus, BD-AChE displays faint fluorescence. Then, the dimethyl carbamate in BD-AChE is recognized by AChE, and the ester bond is cleaved, resulting in BD-AChE being transformed to free BODIPY with fluorescence enhancement. The reaction mechanism is displayed in Scheme 1. Utilizing BD-AChE, we monitored the change of AChE in the brains of mice aging models in real time. Furthermore, in association with BD-AChE, the physiological function of AChE in the brains of aging mice with DR was investigated.

\subsection{Spectroscopic properties}

Under analogous physiological conditions (10 mM HEPES buffer, pH 7.4, and 0.5\% Tween 80), we measured the absorption and fluorescence spectra of BD-AChE $(10 \mu \mathrm{M})$. As displayed in Fig. S1 (ESI $\dagger$ ), the maximum absorption wavelength of $\mathrm{BD}$ AChE was at $693 \mathrm{~nm}\left(\varepsilon_{693 \mathrm{~nm}}=3.42 \times 10^{4} \mathrm{M}^{-1} \mathrm{~cm}^{-1}\right)$. After adding AChE (20.0 $\left.\mathrm{U} \mathrm{mL}^{-1}\right)$, the maximum absorption peak changes to $710 \mathrm{~nm}\left(\varepsilon_{710 \mathrm{~nm}}=4.79 \times 10^{4} \mathrm{M}^{-1} \mathrm{~cm}^{-1}\right)$. We performed the fluorescence titration of $\mathrm{BD}-\mathrm{AChE}$ in the presence of AChE in a concentration range of 0-20.0 $\mathrm{U} \mathrm{mL}^{-1}$. In Fig. 1a, the corresponding fluorescence emission profiles increased with peaks centered at $740 \mathrm{~nm}$. The quantum yield of BD-AChE increased, ranging from 0.03 to 0.27 . We detected the excitation and emission wavelengths of BD-AChE in the NIR range, indicating that $\mathrm{BD}-\mathrm{AChE}$ had the advantage of reducing background interference, which could greatly accelerate the detection sensitivity. In the range of $0-20.0 \mathrm{U} \mathrm{mL} \mathrm{m}^{-1}$, the fluorescence response of $\mathrm{BD}-\mathrm{AChE}$ and $\mathrm{AChE}$ was linearly concentration dependent (Fig. 1b). The equation gained from the calibration curve was $F_{740 \mathrm{~nm}}=19.73[\mathrm{AChE}] \mathrm{U} \mathrm{mL}^{-1}+19.92$ $(r=0.9940)$. The detection limit toward AChE was calculated to be $0.21 \mathrm{U} \mathrm{mL}^{-1}(3 \sigma / k)$, which demonstrated that the probe BDAChE had great sensitivity towards AChE under simulated environments. The ability of our probe as an effective chemical tool to qualitatively and quantitatively detect AChE has been demonstrated by the above results.

\subsection{Kinetic investigation of BD-AChE toward AChE}

With the assistance of the synthesized probe, we assessed the response of $\mathrm{BD}-\mathrm{AChE}$ toward $\mathrm{AChE}$ under analogous experimental conditions. In Fig. 1c, different concentrations of BD-AChE (0.05, 0.10, 1.0, 5.0, 10.0, 20.0, 40.0, 50.0 and 100.0 $\mu \mathrm{M})$ were added into the AChE solution $\left(20.0 \mathrm{U} \mathrm{mL}^{-1}\right)$. With the increase in $\mathrm{BD}-\mathrm{AChE}$ concentration, the reaction rate was accelerated, demonstrating that enzyme activity was the factor which limited the reaction rate. The fluorescence intensity at $1 \mathrm{~min}$ was chosen as the basis to quantify the initial rate of reaction. The kinetic parameters, including the Michaelis constant $\left(K_{\mathrm{m}}\right)$ and maximum initial reaction rate $\left(V_{\max }\right)$, for the enzymatic cleavage reaction of BD-AChE were computed to be $85 \mu \mathrm{M}$ and $5.78 \mu \mathrm{M} \min ^{-1}$ (Fig. 1c), separately. The results proved that the probe has a good response towards AChE. The catalytic activity of AChE towards BD-AChE was also evaluated. Higher concentrations

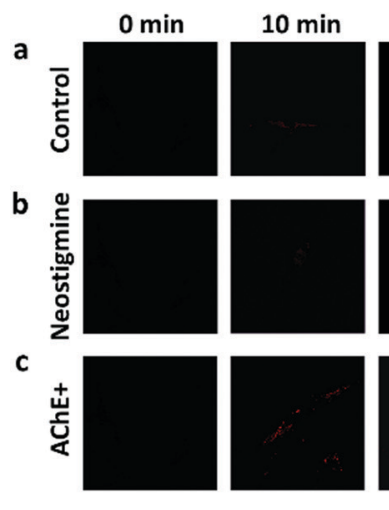

e

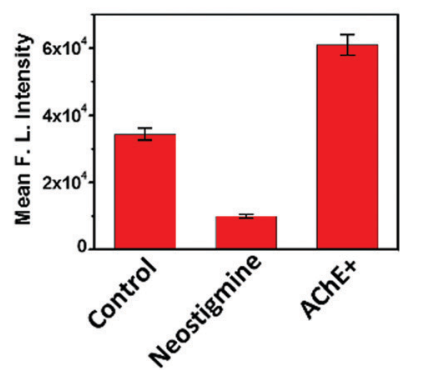

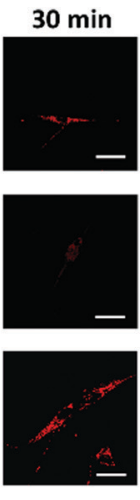

f
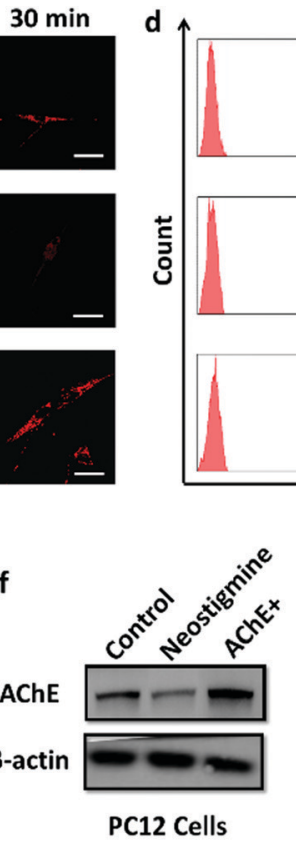
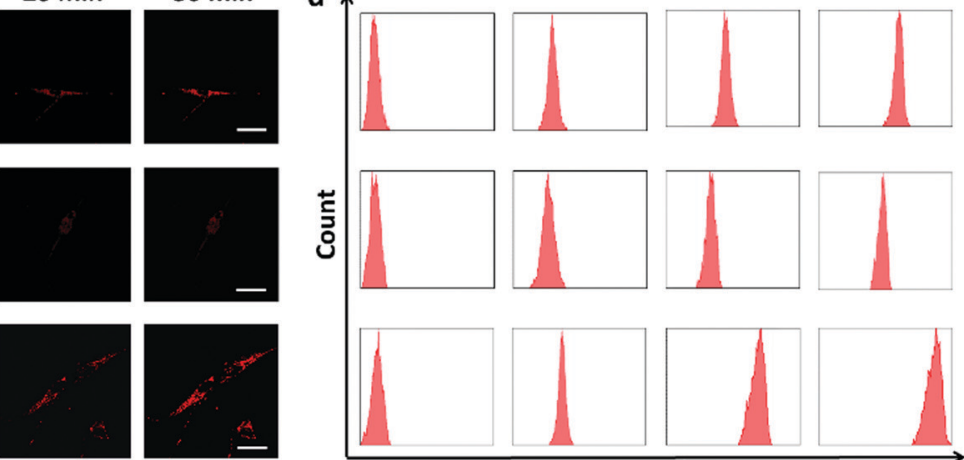

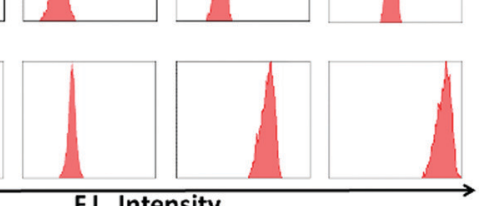

g

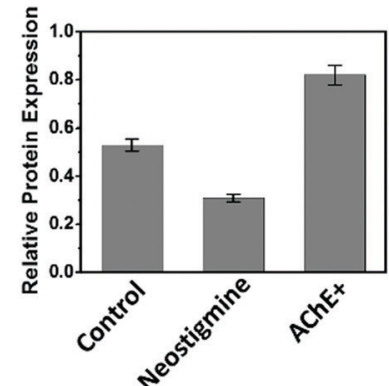

Fig. 2 Confocal microscopy images and flow cytometry assay of PC12 cells for measuring AChE levels in living cells. (a) AChE imaging with BD-AChE in PC12 cells at different time points: 0,10,20, and 30 min via confocal laser-scanning microscope as the control. (b) Prior to being treated as described for the control, PC12 cells were performed with pretreatment of $10 \mu \mathrm{M}$ neostigmine for 30 minutes. (c) PC12 cells were treated as described for the control after AChE transfection. (d) Flow cytometry analysis of $(a-c)$. (e) Mean fluorescence intensity of PC12 cells in (a-c). (f) Western Blot analysis of AChE changes in PC12 cells. We employed $\beta$-actin as the internal control. (g) Quantitative analysis of AChE expression in (f). Red channel: $\lambda_{\text {ex }}=680 \mathrm{~nm}$, $\lambda_{\mathrm{em}}=720-780 \mathrm{~nm}$. The data are displayed as mean (s.d.). Scale bars: $20 \mu \mathrm{m}$. 
of AChE induced a faster response and a greater fluorescence increase, which was proved by studying the fluorescence kinetic curves of BD-AChE with different concentrations of AChE $(0,2.0$, and $20.0 \mathrm{U} \mathrm{mL}^{-1}$ ) for $30 \mathrm{~min}$ (Fig. 1d). When the AChE concentration was $20.0 \mathrm{U} \mathrm{mL}^{-1}$, the fluorescence intensity could reach the plateau within $15 \mathrm{~min}$. These results clarified that BD-AChE responded very quickly to AChE. For further exploring AChE levels in complex biological environments, the real-time imaging capability of BD-AChE for AChE is indispensable.

\subsection{Selectivity of BD-AChE towards AChE}

Next, we measured the fluorescence response of BD-AChE toward other biologically relevant species. As illustrated in Fig. 1e, the probe was incubated with many enzymes (butyrylcholine esterase, pepsin, peroxidase, horseradish, glutathione S-transferases, nitroreductase, monoamine oxidase A, monoamine oxidase B, and alkaline phosphatase), BSA, glucose, and biospecies (cysteine, alanine, glycine, lysine and arginine). The experimental results obviously revealed that a significant increase in fluorescence emission can only be observed in the presence of AChE, and neither common metal ions $\left(\mathrm{Na}^{+}, \mathrm{Ca}^{2+}\right.$, $\mathrm{K}^{+}, \mathrm{Mg}^{2+}$, and $\left.\mathrm{Zn}^{2+}\right)$ nor anions $\left(\mathrm{Cl}^{-}, \mathrm{Br}^{-}, \mathrm{HSO}_{3}{ }^{-}, \mathrm{SO}_{4}^{2-}, \mathrm{SO}_{3}^{2-}\right.$, $\mathrm{S}_{2} \mathrm{O}_{3}^{2-}, \mathrm{CO}_{3}^{2-}, \mathrm{H}_{2} \mathrm{PO}_{4}^{2-}$ ) triggered conspicuous fluorescence signals (Fig. 1f). In conclusion, these results validated that
BD-AChE had excellent selectivity towards AChE and it is an ideal and effective tool for sensitively detecting AChE levels under sophisticated biological systems.

\subsection{Imaging of $\mathrm{AChE}$ in living cells}

Since BD-AChE displayed excellent selectivity towards AChE, we next utilized BD-AChE to detect AChE in living neural cells. The representative PC12 cells were chosen as the testing models. Before the various cell experiments, we applied MTT assay to assess the cytotoxicity of BD-AChE in PC12 cells. The experimental results broadly illustrated that BD-AChE showed less cytotoxicity to PC12 cells under physiological environments (Fig. S2, ESI $\dagger$ ).

Using laser scanning confocal microscopy, we performed cell imaging experiments with BD-AChE. Prior to fluorescence imaging, all tested PC12 cells were incubated with BD$\mathrm{AChE}$ at $37^{\circ} \mathrm{C}$ for $30 \mathrm{~min}$. We then employed flow cytometry to reveal the variation in fluorescence intensity. The tested PC12 cells were divided into three groups separately, as displayed in Fig. 2. The PC12 cells without any treatment as the control are shown in Fig. 2a. Fig. 2b shows the PC12 cells that were pretreated with neostigmine, which is a highefficiency AChE inhibitor and is used in clinics at present. The PC12 cells shown in Fig. 2c were transfected with AChERNA to overexpress AChE. Within the detection time of 30 minutes, the fluorescence of the control group gradually a

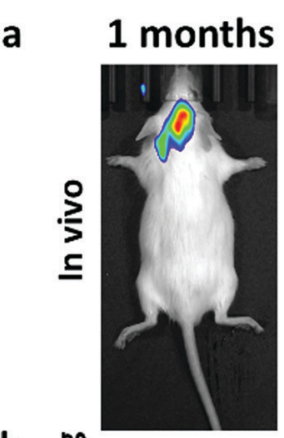

b
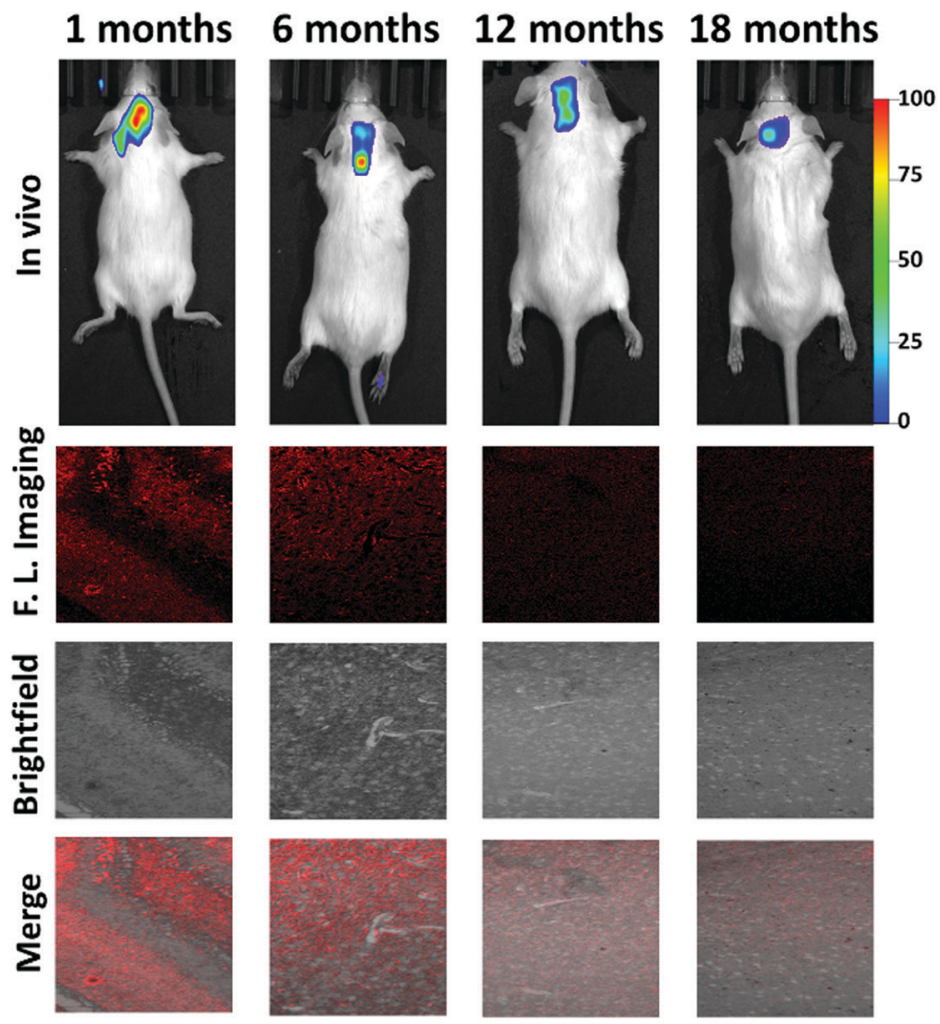

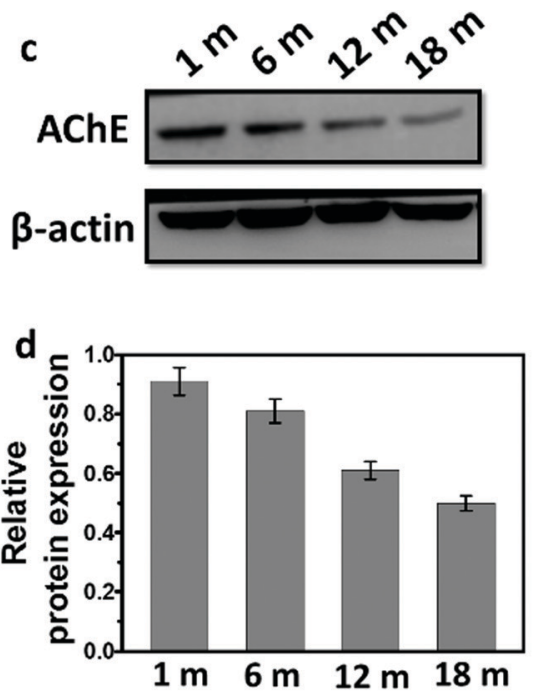

e

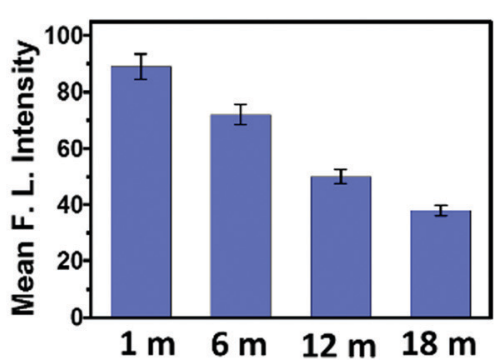

Fig. 3 Imaging of AChE levels in the brains of mice aging models. (a) Imaging of AChE levels in mice brains according to distinct ages: 1, 6, 12 and 18 months old. The mice in the four groups were pretreated with intracranial injection of BD-AChE for 30 min. (b) Imaging of brain slices of mice aging models. (c) Western blot analysis of AChE variation of brain tissue in distinct ages. (d) Quantitative analysis of AChE expression in (c). (e) Mean fluorescence intensity of the images in (a). Fluorescence imaging channel: $\lambda_{\mathrm{ex}}=680 \mathrm{~nm}$, and $\lambda_{\mathrm{em}}=720-780 \mathrm{~nm}$. Data are displayed as mean $\pm \operatorname{SD}(n=5)$. 
a

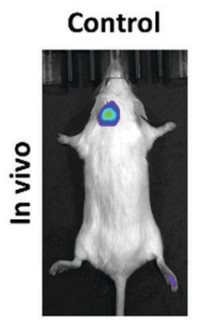

b
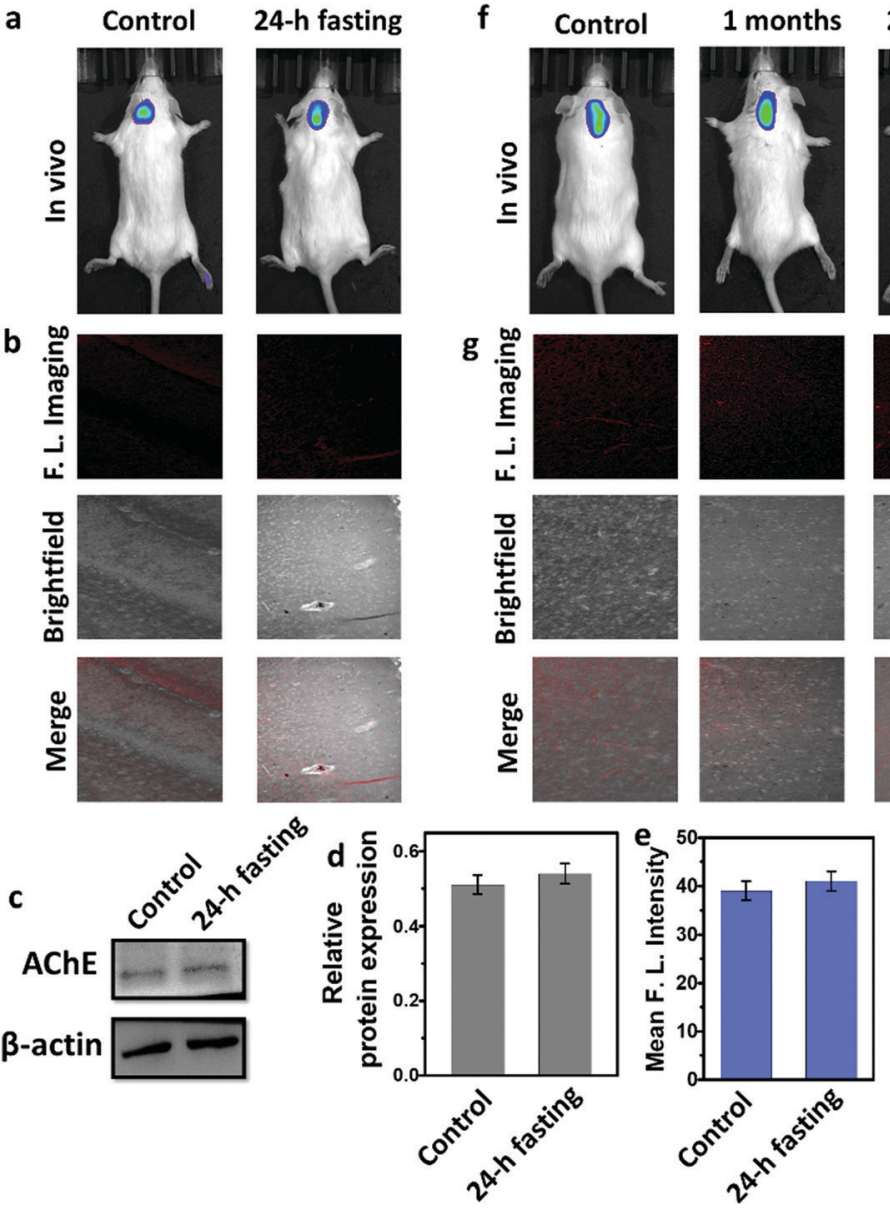
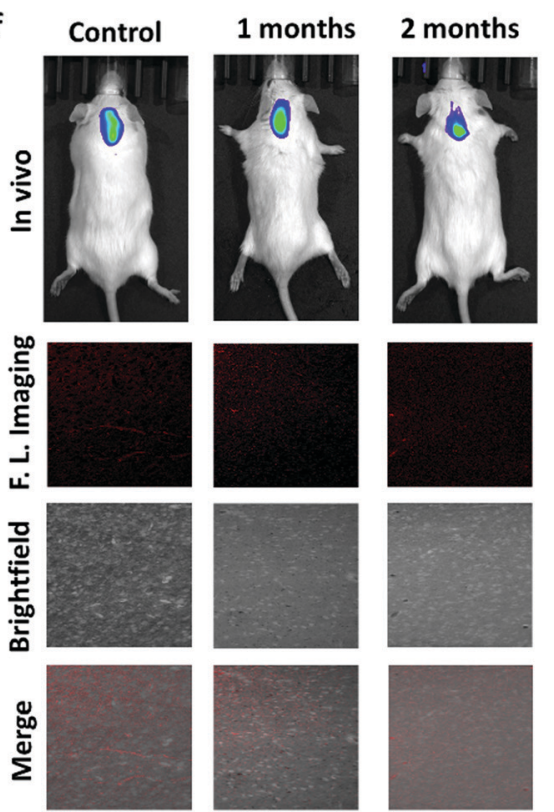

h
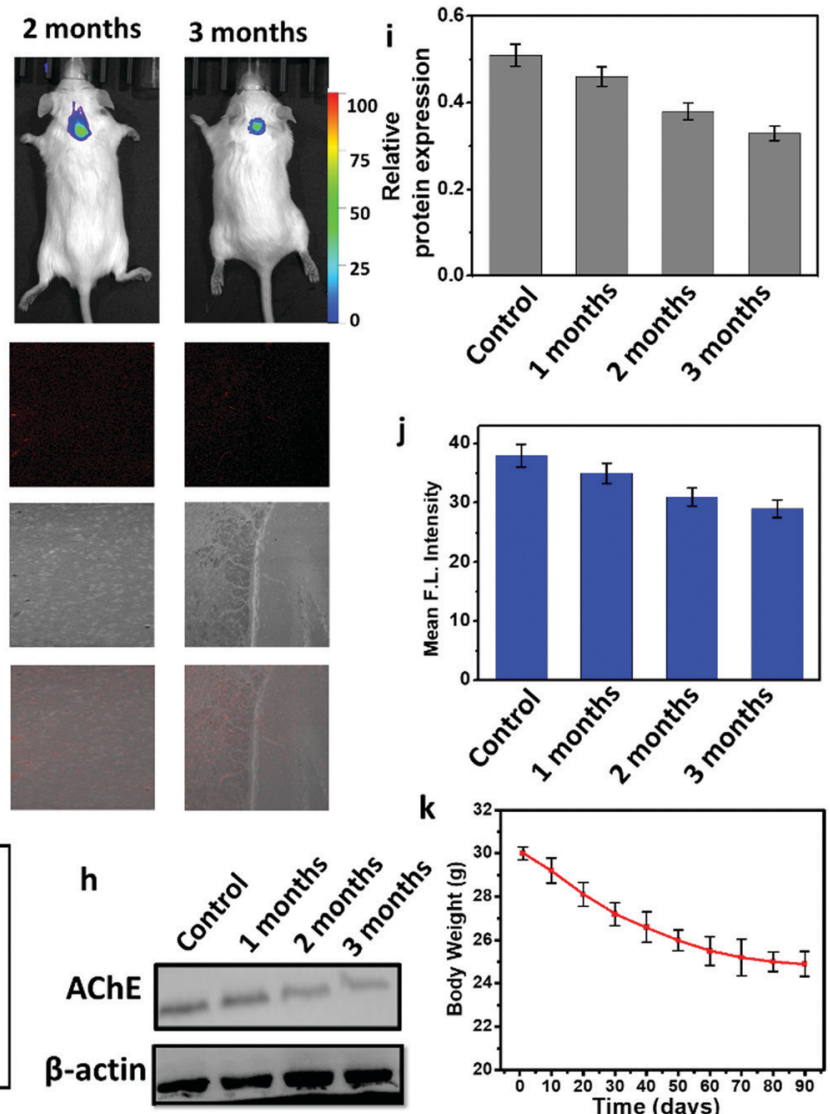

k

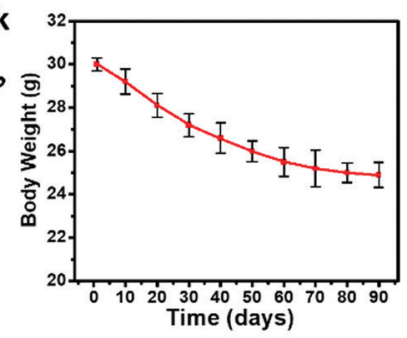

Fig. 4 Effects of DR on the AChE levels in the brains of aging mice. (a) Imaging of AChE levels in the mice brains after short-term $24 \mathrm{~h}$ fasting. The aging mice were treated with a continuous supply of food as a control, while the mice in the $24 \mathrm{~h}$ fasting group were fasted for $24 \mathrm{hours}(24 \mathrm{~h}$-fasted). All the aging mice were treated with BD-AChE by intracranial injection for 30 min. (b) Imaging of the brain slices of (a). (c) Western Blot analysis of AChE variation in the brain tissue of (a). (d) Quantitative analysis of AChE expression in (c). (e) Mean fluorescence intensity of the images in (a). (f) Imaging of AChE levels in the mice brains after long-term DR. Aging mice treated with a continuous supply of food were used as the control group, while the mice under DR were only fed on alternate days for 1, 2 and 3 months, respectively. (g) Imaging of the brain slices of (f). (h) Western blot analysis of AChE variation of the brain tissue in (f). (i) Quantitative analysis of AChE expression in (h). (j) Mean fluorescence intensity of the images in (f). (k) The body weight changes of the aging mice in the DR 3 months group. Fluorescence imaging channel: $\lambda_{\mathrm{ex}}=680 \mathrm{~nm}$, and $\lambda_{\mathrm{em}}=720-780 \mathrm{~nm}$. Data are presented as mean $\pm \mathrm{SD}(n=5)$.

increased. Compared to Fig. 2a, the fluorescence observed in Fig. $2 \mathrm{~b}$ is much weaker, illustrating that the AChE level of PC12 cells in Fig. 2b is lower. Due to the higher AChE level in PC12 cells, a stronger fluorescence is observed in Fig. 2c. The bright field of Fig. $2 \mathrm{a}-\mathrm{c}$ are displayed in Fig. S3 (ESI $\dagger$ ). To further verify the fluorescence variation of the three groups of cells, a flow cytometry assay was employed as shown in Fig. 2d. Fig. 2e displays the mean fluorescence intensity of PC12 cells. These results displayed satisfactory consistency to the fluorescence image results. Besides, we checked the AChE levels in the three groups of PC12 cells using western blotting (Fig. $2 \mathrm{f}$ and g). All results confirmed that BD-AChE was capable of being utilized as an effective and promising tool for the rapid detection of AChE in living cells.

\subsection{Level variation of $\mathrm{AChE}$ in the brains of mice aging models}

Having confirmed that the level of AChE in cells could be tracked using BD-AChE, we next employed BD-AChE to explore the fluctuation of AChE levels in living mice, particularly in the brain. Before exploring the potential relationship between the level of AChE and DR in old mice, we first examined the changes in the level of AChE in cell aging models. Based on the difference in age: 1, 6, 12 and 18 months old, we divided the mice models into four groups. Before imaging, the mice in the four groups were pretreated by intracranial injection of BD-AChE. The fluorescence intensity of the brain became weaker as the age increased (Fig. 3a), clarifying that the AChE level decreased with age. The mean fluorescence intensity is displayed in Fig. 4e. The fluorescence imaging of the brain slices (Fig. 3b) and western blot (Fig. 3c and d) were performed for further evaluating the fluctuation in AChE levels. These results not only demonstrated that BD-AChE can be potentially utilized to detect the AChE level in vivo, but also revealed the fact that the AChE level decreased with increase in age. 


\subsection{Effect of DR on the level of AChE}

On the basis of the theory of hormesis mechanism, iterative slight stress has a positive impact from the cell responses and the repair and maintenance processes stimulated by repeated exposure to stress results in aging-retardant action. ${ }^{34-36} \mathrm{DR}$ is a cumulative slight stressor which improves the body's capability in coping with serious stressors by increasing cellular metabolic adjustments, stress resistance and adaptability. ${ }^{37}$ Recent studies focused on the mechanism by which DR provides its benefits. Research studies confirm that DR is an intervention that protects the body against protein misfolding diseases, and ER hormesis is used as a major mechanism of DR function. ${ }^{38}$

Encouraged by the satisfying function of BD-AChE in specifically detecting and imaging endogenous AChE in cells and in vivo, we next attempted to explore the effect of DR on AChE levels in the brains of aging mice. Before detecting the effects of long-term DR on AChE, we first detect how AChE changes after a short $24 \mathrm{~h}$ fasting. We divided the old (18 months) mice in our studies into two groups. The first group is the ad libitum fed control group with a continuous supply of food, while the mice in the second group were fasted for 24 hours ( $24 \mathrm{~h}$-fasted). However, we regularly supplied water to all the groups. Before imaging, all the tested mice were pretreated with intracranial injection of BD-AChE. As shown in Fig. 4a, no significant fluorescence fluctuation in the brain was observed in the two groups. Fig. 4e displays the mean fluorescence intensity of Fig. 4a. The fluorescence imaging of brain slices was performed to further confirm the level of AChE, as was western blot (Fig. 4b-d). These results indicated that a $24 \mathrm{~h}$ short-term fasting had no obvious effect on AChE in the brain of aging mice. Then, we investigated whether the level of AChE changed after long-term DR. The first group was the control. In the other group, aging mice under the DR regimen were only fed on alternate days for 1, 2 and 3 months, respectively. In Fig. $4 \mathrm{f}$, the fluorescence intensity in the DR groups was much weaker than that in the control group, and the fluorescence degrees in the DR groups was as follows: 1 months $>2$ months $>3$ months. We also acquired the fluorescence imaging of the brain slices, as well as western blot data (Fig. $4 \mathrm{~g}-\mathrm{i}$ ). In the data we obtained, we noticed that the body weight of aging mice decreased during long-term DR (3 months) (Fig. 4k). These results revealed the intimate connection between DR and AChE, and the level of AChE decreased after long-term DR. Our research displayed that DR had a more pronounced and long-lasting effect on AChE compared to $24 \mathrm{~h}$ fasting.

\section{Conclusions}

In conclusion, by concentrating on the part of DR in the regulation of AChE in the brains of aging animals, we designed a probe BD-AChE for detecting AChE variation in living cells and in vivo with excellent selectivity and sensitivity, particularly in the brain. Our experimental results validated that the AChE levels decreased in the brains of mice aging models. Notably, the AChE levels in the brains of aging mice significantly decreased after DR. With the assistance of BD-AChE, in vivo visualization for the first time displayed the significant impact of DR on AChE in the brains of aging mice, which helps to provide a natural alternative to treating a variety of age-related neurodegenerative diseases without the use of exogenous drugs.

\section{Conflicts of interest}

There are no conflicts to declare.

\section{Acknowledgements}

We thank the National Nature Science Foundation of China (No. 81573393, 21804010, 21976209, 22007005), the Science and Technology Innovation Development Plan of Yantai of China (No. 2020MSGY113), the Research Initiation Fund of Binzhou Medical University (Grant No. BY2019KYQD39, BY2020KYQD01), Taishan Scholar Project Special Funding (No. ts20190962), the Shandong Peninsula Engineering Research Center of Comprehensive Brine Utilization (2018LS014), and Weifang Science and Technology Development Project (2019GX076).

\section{References}

1 E. Perry, J. Court, R. Goodchild, M. Griffiths, E. Jaros, M. Johnson, S. Lloyd, M. Piggott, D. Spurden, C. Ballard, I. McKeith and R. Perry, J. Neural Transm., 1998, 105, 915-933.

2 Y. G. Prall, K. K. Gambhir and F. R. Ampy, Life Sci., 1998, 63, 177-184.

3 J. A. Dumas and P. A. Newhouse, Pharmacol., Biochem. Behav., 2011, 99, 254-261.

4 R. T. Bartus, R. R. Dean, B. Beer and A. S. Lippa, Science, 1982, 217, 408-414.

5 P. Anand and B. Singh, Arch. Pharmacal Res., 2013, 36, 375-399.

6 A. J. Franjesevic, S. B. Sillart, J. M. Beck, S. Vyas, C. S. Callam and C. M. Hadad, Chemistry, 2019, 25, 5337-5371.

7 E. J. Masoro, Interdiscip. Top. Gerontol., 2007, 35, 1-17.

8 S. Ribaric, Oxid. Med. Cell. Longevity, 2012, 2012, 741468.

9 J. R. Speakman and S. E. Mitchell, Mol. Aspects Med., 2011, 32, 159-221.

10 N. Pitsikas and S. Algeri, Neurobiol. Aging, 1992, 13, 369-373.

11 Z. F. Yu and M. P. Mattson, J. Neurosci. Res., 1999, 57, 830-839.

12 C. M. Hine and J. R. Mitchell, J. Clin. Exp. Psychopathol., 2012, S4.

13 J. Su, H. Liu, K. Guo, L. Chen, M. Yang and Q. Chen, Molecules, 2017, 1, 176-199.

14 G. L. Ellman, K. D. Courtney, V. J. Andres and R. M. FeatherStone, Biochem. Pharmacol., 1961, 7, 88-95.

15 X. Ren, J. Wei, J. Ren, L. Qiang, F. Tang and X. Meng, Colloids Surf., B, 2015, 125, 90-95.

16 S. Hou, Z. Ou, Q. Chen and B. Wu, Biosens. Bioelectron., 2012, 33, 44-49. 
17 V. Pavlov, Y. Xiao and I. Willner, Nano Lett., 2005, 5, 649-653.

18 V. Marx, Nat. Methods, 2014, 11, 717-720.

19 R. Wang, C. Yu, F. Yu and L. Chen, TrAC, Trends Anal. Chem., 2010, 29, 1004-1013.

20 Y. Yang, Q. Zhao, W. Feng and F. Li, Chem. Rev., 2012, 113, 192-270.

21 X. Li, X. Gao, W. Shi and H. Ma, Chem. Rev., 2013, 114, 590-659.

$22 \mathrm{X} . \mathrm{Wu}, \mathrm{H} . \mathrm{Li}, \mathrm{E}$. Lee and J. Yoon, Chemistry, 2020, 11, 2893-2901.

23 X. Chen, Y. Zhou, X. Peng and J. Yoon, Chem. Soc. Rev., 2010, 39, 2120-2135.

24 D. Wu, A. C. Sedgwick, T. Gunnlaugsson, E. U. Akkaya, J. Yoon and T. D. James, Chem. Soc. Rev., 2017, 46, 7105-7123.

25 J. Fan, M. Hu, P. Zhan and X. Peng, Chem. Soc. Rev., 2013, 42, 29-43.

26 J. T. Hou, W. X. Ren, K. Li, J. Seo, A. Sharma, X. Q. Yu and J. S. Kim, Chem. Soc. Rev., 2017, 46, 2076-2090.

27 F. Feng, Y. Tang, S. Wang, Y. Li and D. Zhu, Angew. Chem., Int. Ed., 2007, 46, 7882-7886.

28 D. Liao, J. Chen, H. Zhou, Y. Wang, Y. Li and C. Yu, Anal. Chem., 2013, 85, 2667-2672.
29 S. Liao, W. Han, H. Ding, D. Xie, H. Tan, S. Yang, Z. Wu, G. Shen and R. Yu, Anal. Chem., 2013, 85, 4968-4973.

30 Y. Zhang, Y. Cai, Z. Qi, L. Lu and Y. Qian, Anal. Chem., 2013, 85, 8455-8461.

31 H. Tong, Y. Hong, Y. Dong, M. Haussler, J. W. Lam, Z. Li, Z. Guo, Z. Guo and B. Z. Tang, Chem. Commun., 2006, 3705-3707.

32 X. Wang, P. Li, Q. Ding, C. Wu, W. Zhang and B. Tang, J. Am. Chem. Soc., 2019, 141, 2061-2068.

33 X. Wu, J. M. An, J. Shang, E. Huh, S. Qi, E. Lee, H. Li, G. Kim, H. Ma, M. S. Oh, D. Kim and J. Yoon, Chem. Sci., 2020, 11, 11285-11292.

34 G. E. Guo, L. W. Ma, B. Jiang, J. Yi, T. J. Tong and W. G. Wang, J. Cell. Biochem., 2010, 109, 1000-1005.

35 S. Haider, S. Saleem, T. Perveen, S. Tabassum, Z. Batool, S. Sadir, L. Liaquat and S. Madiha, Age, 2014, 36, 9653.

36 S. I. Rattan, J. Gerontol., Ser. A, 2004, 59, 705-709.

37 M. P. Mattson, Ageing Res. Rev., 2008, 7, 43-48.

38 L. Matai, G. C. Sarkar, M. Chamoli, Y. Malik, S. S. Kumar, U. Rautela, N. R. Jana, K. Chakraborty and A. Mukhopadhyay, Proc. Natl. Acad. Sci. U. S. A., 2019, 116, 17383-17392. 